\title{
Ciencia y arte: Investigación empleando medios artísticos
}

\author{
Dra. Hilda WENGROWER ${ }^{1}$ \\ mshilda@mscc.huji.ac.il
}

Recibido: 03/07/12

Aceptado: 22/11/12

\section{RESUMEN}

Se presenta la necesidad de impulsar la investigación de las terapias a través de las artes, aceptando la posibilidad del empleo de metodologías mixtas (cualitativas y cuantitativas) a fin de lograr una práctica basada en la evidencia. El artículo enfatiza la investigación empleando medios artísticos y trae ejemplos.

Palabras clave: Investigación por medio de las artes. Terapias a través de las artes. Práctica basada en la evidencia.

\section{Referencia normalizada}

Wengrower H. (2012). "Ciencia y arte: Investigación empleando medios artísticos". En Arteterapia: Papeles de arteterapia y educación artística para la inclusión social Vol.7: páginas 41-56. Madrid. Servicio de publicaciones UCM.

\section{SUMARIO}

Introducción. La necesidad de impulsar la investigación en las terapias creativas a través de las artes. Paradigmas de investigación. Diferencias entre las demandas de la investigación cuantitativa y práctica clínica. Las artes como medio de construcción de conocimiento en investigación. ¿Cuándo es apropiado investigar con medios artísticos? Validez y confiabilidad. Algunas técnicas para asegurar confiabilidad y validez. Algunos modos de empleo de técnicas artísticas en investigación: Etnodrama o performance etnográfica. Danzando la información. Exploración de experiencias y temas relacionados con género por medio de artes escénicas. Empleo de técnicas de dramaterapia para investigación psicosocial. Conclusión

\section{ABSTRACT}

\section{Science and Art: Research using artistic means}

The paper encourages research in Arts Therapies, accepting mixed methodologies and emphasizing arts based research in order to achieve an evidence based practice. Some examples are brought.

Keywords: Arts based research. Arts therapies. Evidence based practice.

\section{CONTENTS}

Introduction. The need of increasing research in arts therapies. Paradigms in research. Differences between quantitative research demands and clinical practice.

The arts as a means to construct knowledge. When is it adequate to employ art based research? Validity and reliability. Some techniques to assure reliability and validity. Examples of the arts based re-

${ }^{1}$ Univ. Hebrea de Jerusalén, Dep. de estudios de Teatro. Danza Movimiento Terapeuta. Coeditora del libro La Vida es Danza: el Arte y la Ciencia de la Danza Movimiento Terapia (Gedisa) y autora de diversos artículos. Imparte clases y supervisiones en diferentes países. Supervisión a través de Skype. 
search. Ethnodrama. Dancing the data. Exploring sexuality and gender. Dramatherapy in psychosocial research. Conclusion.

\section{INTRODUCCIÓN}

La exigencia de presentar evidencias empíricas sobre la eficacia de las psicoterapias es ya una realidad todos los países, es lo que se llama "práctica basada en la evidencia" (Evidence Based Practice). Esto implica la integración de la pericia y habilidad clínica del profesional con el conocimiento de evidencias producidas por medio de investigaciones experimentales, enmarcadas en el contexto de las características específicas del paciente, sus preferencias y cultura.

Aun cuando la American Psychological Association y otras entidades profesionales han enfatizado el método experimental, en el marco de este artículo nos dedicaremos al aporte de las artes y en especial de las técnicas teatrales o dramáticas, tanto epistemológicamente (el estudio de los modos de construcción del conocimiento) como metodológicamente.

\section{LA NECESIDAD DE IMPULSAR LA INVESTIGACIÓN EN LAS TERA- PIAS CREATIVAS A TRAVÉS DE LAS ARTES}

Freud definió el psicoanálisis como la investigación del inconsciente. Los psicoterapeutas de varias corrientes teóricas y modalidades, ven en la exploración un procedimiento central de su tarea, ya sea del inconsciente, de las motivaciones, las relaciones interpersonales, intrafamiliares, los significados de las vivencias para los sujetos, etc.

Chaiklin y Chaiklin (2004) observan varias analogías entre el trabajo clínico y el de investigación que enuncio a continuación.

Los terapeutas formulan hipótesis acerca de la raíz de los problemas que aquejan a las personas que acuden en su ayuda.

En ocasiones intentan predecir o determinar el curso que pueden tomar aspectos de la vida de los pacientes.

Seleccionan los procedimientos que consideran adecuados a fin de lograr los objetivos terapéuticos que se plantean en beneficio de los pacientes o usuarios.

Recogen y analizan información.

Todas estas operaciones son similares a las que llevan a cabo los científicos.

La investigación ha sido definida como un proceso sistemático y crítico de indagación cuyo objetivo es producir conocimiento fundamentado (Williams e Irving, 1999 en Cruz y Berrol 2004). Estos autores consideran que los terapeutas reticentes a la investigación pueden caer en los siguientes peligros:

A nivel epistemológico, basarse en opiniones o ideas personales cuya validez no puede ser constatada.

Basarse en ideas axiomáticas que excluyen información de fuentes variadas y sólidas.

Falta de rigor o agudeza teórica.

Contrario a lo anterior, adherirse ciegamente a una teoría independientemente de que se hayan hallado evidencias en su favor. 
La investigación satisface necesidades internas a la comunidad de terapeutas y otras que nuestra ubicación en el contexto sociocultural demanda. Estas necesidades son:

Generar hipótesis de trabajo.

Constatar o falsear esas hipótesis o conocimientos.

Enriquecer nuestro pensamiento.

Mejorar habilidades técnico-profesionales.

Monitorizar el proceso terapéutico y/o evaluar cambios.

Comprobar o falsear teorías y técnicas de la profesión.

Verificar y demostrar la eficacia de los tratamientos, compararlos con otras modalidades terapéuticas e identificar su contribución específica.

Lograr reconocimiento y ampliar nuestra presencia en organizaciones de asistencia ya sea en el orden estatal, público o privado.

En terapia a través del movimiento y la danza el avance en investigación es relativo. Se publica, mas la mayoría de las investigaciones son con muestras pequeñas, y no se hacen réplicas.

Hay tres revistas especializadas en inglés: American Journal of Dance Therapy; Body, Movement and Dance in Psychotherapy; y The Arts in Psychotherapy. El nivel en todas ellas es muy bueno. A menudo se presenta el dilema sobre dónde publicar: si en las revistas mencionadas, que se dirigen fundamentalmente a un público entendido en la materia y colega de profesión, o en otras revistas más generales, donde se puede difundir más lo nuestro, pero donde será tal vez más difícil de ser aceptados.

Meekums (2010) menciona una propuesta interesante de Margison y cols. (2000), la red de investigación y práctica (Practice Research Networks o PRNs) que se adecua a terapeutas quienes no están normalmente abocados a la tarea de recolección de datos y selección de patrones acordados previamente. Esta estrategia contribuye a la práctica basada en la evidencia, la cual está recibiendo adhesión como alternativa válida al modelo de investigaciones llevadas a cabo con asignación aleatoria de sujetos en grupo de intervención y grupo de control. Consiste como su nombre lo indica, en organizar una especie de base datos definidos lo más claramente posible, que pueden emplearse para constatar y comparar los resultados de tratamientos terapéuticos.

Un texto hebreo del siglo III dice aproximadamente: Si no me ocupo de lo que me concierne, ¿quién lo hará? Y si sólo me ocupo de lo mío, ¿quién soy? Y si no lo hago ahora entonces ¿cuándo? ( Pirkei Avot 1: 13). Es decir, sólo los terapeutas investigaremos nuestra tarea, por nuestro bien y el de nuestros pacientes. No podemos postergar esta labor.

\section{PARADIGMAS DE INVESTIGACIÓN}

Hace más de 300 años que el modelo positivista empírico domina el pensamiento y la acción científica. Sus objetivos principales son la predicción y el control del fenómeno que es objeto de estudio y la formulación de explicaciones al mismo. Por 
control se entiende destilar los factores que influyen en el fenómeno o en la cuestión que genera el interés de la investigadora y asegurarse que el método empleado y los datos producidos se relacionan fehacientemente.

Paralelamente, la hermenéutica o la indagación interpretativa constituye otro paradigma en el cual lo importante es comprender, relativizar y contextualizar.

La definición y conceptualización de investigación es un fenómeno construido socialmente y por tanto va modificándose históricamente. Hace ya décadas que tiene lugar la puja entre ambos paradigmas y afortunadamente el modelo interpretativo va haciéndose espacio a través de publicaciones científicas, revistas especializadas, asociaciones académicas y congresos.

Siguiendo en el tema del contexto sociocultural en la investigación, me referiré brevemente al aporte de Lenore Hervey. Al reflexionar sobre los factores que estimulan o desalientan la indagación ${ }^{2}$, Hervey considera que es necesario explorar cómo la cultura en la que estamos insertados conceptualiza el arte, la ciencia, la salud, la enfermedad y la curación (Hervey 2008). Esta autora coincide con Elliot Eisner al indicar que el clima político en la comunidad de profesionales de la salud y en los centros científicos, es un factor que también hace mella en este aspecto de nuestro devenir profesional. Asimismo los modos de definir el conocimiento y los medios con los cuales contamos para desarrollarlo inciden en la tarea de la indagación científica.

Pico (1988, en Wengrower y Serrano, 2009) nos recuerda que los artistas se han adelantado a las ciencias en más de una ocasión en la visión-comprensión de la realidad. Las corrientes artísticas del expresionismo, el cubismo, el dadaísmo y el simbolismo, para nombrar algunas, nos pusieron en contacto consciente y vivencial con la multiplicidad de paradojas, ambigüedades, la relatividad, la polisemia y las incertidumbres del acontecer humano.

El paradigma positivista considera que la realidad está a la espera de ser percibida o encontrada. En cambio, el paradigma construccionista concibe la realidad social y humana como algo que las personas, incluidos los científicos, configuran a partir de sus pre-conceptos, características personales, ubicación sociohistórica, medios de aproximación y técnicas de las que disponen. Estamos así frente a una perspectiva que enfatiza el carácter interpretativo del conocimiento que admite la inclusión del arte como medio válido científicamente.

\section{DIFERENCIAS ENTRE INVESTIGACIÓN CUANTITATIVA Y PRÁCTICA CLÍNICA}

La investigación en base a modelos cuantitativos de investigación no proporcionó suficientes guías para la intervención clínica. Los criterios para este tipo de indagación no coinciden con los que gobiernan una buena práctica clínica. En la primera predomina la generalización y en la segunda, sin dejar de lado el conocimiento de

\footnotetext{
${ }^{2}$ En la publicación aludida se trata de la terapia a través de la danza y el movimiento.
} 
características universales, se destaca la atención a lo particular, a lo específico del caso que se trata. Si pensamos en trastornos de baja incidencia en la población, el problema es aún mayor ya que las dificultades para satisfacer las demandas estadísticas incrementan. El mismo DSM (Manual Diagnóstico y Estadístico de Trastornos Mentales) nos muestra que una categoría diagnóstica incluye una variabilidad de síntomas muy grande.

E. Schopler quien ha llevado adelante una extensa tarea de estudio e investigación en el tema del autismo, dice que la efectividad de un tratamiento o de un servicio asistencial no puede juzgarse según métodos experimentales. Éstos suponen una asignación aleatoria de los sujetos que raramente se puede cumplir, dados los riesgos de carácter ético en los que se puede incurrir. Considera que existe una brecha entre la investigación y la clínica. Informa acerca del grupo de tarea organizado en la APA (American Psychological Association) que se propuso salvar esa distancia estableciendo una distinción entre eficacia y efectividad. El primer término se emplea para modelos clínicos constituidos por intervenciones variadas y variables y diagnóstico de comorbilidad. Efectividad se aplica a los tratamientos apoyados por métodos característicos de las ciencias físicas. Los directores de este grupo concluyeron en 1998 que los tratamientos nunca llegan a demostrar ser totalmente efectivos, pero sí eficaces.

J. Detweiler (2000) reseñó un compendio de resultados efectivos en tratamientos farmacológicos y psicosociales. Notó que los reportes de efectividad variaban según se dirigían a educadores, investigadores, consumidores, etc. Concluye que no hay duda de que los tratamientos analizados sean eficaces, pero no por haber cumplido con criterios científicos inalcanzables.

Podemos acogernos a Green y Britten (1998) quienes se suman a las críticas a la medicina basada en evidencia diciendo que medicina (y psicoterapias en nuestro caso) son algo más que la aplicación automática de reglas científicas. La experiencia clínica basada en la observación, reflexión y apreciación o juicio es necesaria para determinar la adecuación a los casos específicos. La investigación cualitativa se relaciona con aspectos a los cuales la epidemiología (estadística) no llega. Esta modalidad explora entre otras cosas, las actitudes, creencias, preferencias, tanto de los pacientes como de los profesionales.

Green y Britten añaden que los métodos cualitativos ofrecen el tan anhelado puente entre la evidencia y la práctica clínica. Su artículo analiza procedimientos médicos, no obstante es muy relevante para nuestro campo. Los resultados cualitativos proveen información sobre los tratamientos en forma contextualizada. Esto no implica de ningún modo un rechazo de metodologías cuantitativas por parte de estos autores, pero relativizan la hegemonía que se intenta adjudicar a dichas metodologías y presentan las contribuciones del pensamiento cualitativo. Más detalladamente, los aportes del modelo cualitativo que despliegan son:

Naturalismo: al llevar a cabo los estudios en su ámbito natural, informan de los aspectos contextuales que hacen al éxito o fracaso de un tratamiento.

Interpretación: los métodos cualitativos distinguen entre información objetiva y el significado subjetivo que ésta tiene para los pacientes y o los profesionales invo- 
lucrados, cómo la interpretan. Con información objetiva se refieren a análisis, tests, etc.

Proceso: a mi criterio este es un aspecto que también los métodos cuantitativos se proponen seguir, la continuidad de los efectos del tratamiento. Esto se manifiesta en el procedimiento de test-postest y follow up. Cualitativamente la contextualización y la interpretación del factor tiempo es fundamental. ${ }^{3}$

Interacción y relativismo (de factores y de información): también este es un aspecto común a ambas metodologías, pero en la cualitativa es inherente a su conceptualización del mundo y los seres humanos (ontología) y del conocimiento (epistemología).

Estos autores como tantos otros y como es ya más aceptado, señalan que la elección metodológica debe basarse en el carácter de la pregunta que motiva la investigación. Actualmente se recomienda el empleo de métodos mixtos, cuantitativos y cualitativos. De este modo se puede lograr información que pueda constituir evidencia generalizable a otros casos, transmitir el carácter sutil y especial de las terapias a través de las artes, la experiencia por la que pasan los pacientes o usuarios. La metodología mixta también permite observar en profundidad el proceso de la intervención profesional, saber cómo los usuarios vivencian y entienden su experiencia. El desafío consiste entonces en confeccionar un diseño de investigación y una transmisión de la información producida que sea convincente y pueda ser leídapercibida sin ser engorrosa.

Pasaremos a enfocarnos específicamente en el uso de medios artísticos en la investigación.

\section{LAS ARTES COMO MEDIO DE CONSTRUCCIÓN DE CONOCIMIENTO EN INVESTIGACIÓN}

La comprensión de la experiencia humana expresada por medios artísticos no sólo es parte fundamental de las terapias por medio de las artes, sino que esta tarea puede contribuir al conocimiento en sectores afines, como la psicología social, la sociología, la antropología, etc (Wengrower y Serrano, 2009).

La mayoría de los lectores de la revista Arteterapia concordarán en que las palabras no son suficientes para expresar o acceder a los recovecos del alma humana. M. Polany (en Wengrower y Serrano, 2009), propone el concepto de conocimiento tácito, ya que considera que las personas saben más de lo que pueden decir verbalmente. No se refiere solamente a aspectos inconscientes, sino que el lenguaje verbal nunca presenta información completa. Además, hace décadas que los estudios de comunicación verbal han destacado el altísimo componente de expresión no verbal en la interacción interpersonal, las acciones de las personas también son información y sabemos que las artes implican un componente de acción. Tenemos entonces

\footnotetext{
${ }^{3}$ Green y Britten consideran que los aspectos 3 y 4 son específicos de la investigación cualitativa. Por mi parte creo que es necesario matizar su aseveración, del modo en el que lo presento.
} 
otra justificación para la incorporación de medios expresivos no verbales.

Hervey (2004) define la indagación artística en tres modalidades: 1) El empleo de métodos artísticos de colección, análisis y/o presentación de datos, 2) el uso, reconocimiento y análisis del proceso creativo y 3 ) la exploración motivada y determinada por los valores estéticos de quien se embarca en ella.

Los medios artísticos pueden ser usados en los diferentes pasos de una investigación:

en la recolección o producción de datos, en el análisis o en la presentación final.

La metodología cualitativa es consistente con los principios y métodos de las terapias creativas, constituye una integración sistemática y exhaustiva de los métodos empíricos desde una perspectiva semántica básica de este término, ya que se basan en los sentidos y de los métodos introspectivos (Mc Niff,1998). Su núcleo es la comprensión de lo complejo y lo ambiguo apoyándose en la empatía, en la percepción del sujeto con quien se investiga, elementos clave en la tarea terapéutica. Al emplear esta denominación y no la de sujeto de la investigación manifiesto otro aspecto de la investigación cualitativa, su carácter participativo y respetuoso de las personas que toman parte en el proceso de construcción de conocimiento. Asimismo la metodología cualitativa reconoce el papel decisivo y participante del investigador como contrapartida al modelo del investigador neutral (Wengrower y Serrano 2009).

Si se investiga algo relacionado con la tarea arteterapéutica, el empleo de técnicas artísticas mantendrá con mayor fidelidad el espíritu del trabajo realizado.

Los allegados al arte, saben que el contenido está in-corporado en la forma. En el caso de terapia a través de la danza y el movimiento y en teatro-terapia o dramaterapia (como se denomina en inglés), estamos frente a lenguajes semejantes, en cuanto a que forma y contenido se significan y representan: una postura erguida es muy diferente a una postura encorvada desde la perspectiva tanto semántica como estética y desde las experiencia del observador y el observado (Wengrower, 2008; 2009). Por tanto, dependiendo de las técnicas de investigación empleadas, en algunas ocasiones estamos en peligro de perder información tanto en la etapa de recogerla o al transmitirla verbalmente y más aun en escrito. Si investigamos las relaciones intergrupales (identidades colectivas diferentes), el uso que hacen los participantes del espacio en el grupo que se observa o en el cual se interviene, puede ser tanto o más significativo que el discurso verbal: se puede estar usando una expresión verbal que enfatiza la cercanía entre los miembros de los grupos, cuando en realidad están espacialmente distanciados. O sea que la armonía o el contraste entre la expresión hablada, la gestual y el empleo del espacio, brindan material de suma importancia.

\section{¿CUÁNDO ES APROPIADO INVESTIGAR CON MEDIOS ARTÍSTICOS?}

1. Cuando un tema ha sido poco estudiado o definido: los métodos cualitativos y entre ellos los artísticos ofrecen la posibilidad de una indagación exploratoria, descriptiva, a partir de la cual se podría a posteriori establecer preguntas delimitadas. Éstas incluso podrían ser examinadas cuantitativamente.

Ejemplo: hasta comienzos de la década del 1990, la mayoría de las investigaciones llevadas a cabo sobre la integración social de los niños inmigrantes se basaban 
en estadísticas y/o en teorías sociocognitivistas. No había descripciones de la vivencia subjetiva de los niños involucrados, ni mucho menos una conceptualización de este tema como una interacción. Una investigación llevada a cabo con grupos en tres escuelas de educación primaria e intermedia en Jerusalén que incluyó ocho sesiones de expresión y juego teatral para cada grupo, permitió recoger información acerca de este aspecto desde una perspectiva psicodinámica (Wengrower, 1992).

2. Cuando la información que se busca forma parte de un contexto imbricado de modo tal que cualquier manipulación de variables provoca cambios cardinales en la experiencia y la información que produce. Acá influyen las perspectivas ontológica y epistemológica que el investigador adopta. El mismo fenómeno puede ser conceptualizado de modo más o menos abarcador según el paradigma metateórico que constituye el marco más amplio de pensamiento.

Ejemplo: en 1999-2000 llevé a cabo una etnografía en una escuela que se propuso dar respuesta a las siguientes preguntas:

1. ¿Cuál es la figura que los niños autóctonos elaboran de los inmigrantes?

2. ¿Cómo se sienten los inmigrantes en el entramado social de la escuela?

3. ¿Qué tipo de relaciones se establecen entre ellos?

4. ¿Cuáles son las manifestaciones de la reproducción interpretativa que los niños practican?

Partí de una perspectiva socioconstructivista y del paradigma de la Complejidad de E. Morin, ambas conciben los fenómenos psicosociales de forma imbricada y conteniendo niveles diversos de realidad y de factores intervinientes. Por consiguiente, elegí la etnografía como metodología amplia que permite observar un fenómeno en su contexto natural atendiendo a aspectos macro y microsociales. Además de la observación participante diversos procedimientos fueron incorporados: lectura de documentos oficiales, entrevistas grupales a los niños, asociación libre de palabras y dos grupos de juegos teatrales. Cada técnica ofreció información específica (Wengrower y Serrano 2009).

3. Cuando el objeto de la investigación se relaciona con sentimientos, actitudes, relaciones y el cometido es dar luz a expresiones ricas en registro emocional, es claro que las técnicas que nos ocupan son las más adecuadas. Si nos interesan las relaciones interculturales o intergrupales por ejemplo, los factores emocionales están tan presentes y activos como los discursivos, cognitivos, etc., tanto en niños como en adultos. Eiser (1989) comentó críticamente la paradoja creada en la investigación en este campo: la psicología había estado investigando algo tan poco racional como las actitudes con técnicas basadas en principios que sólo atienen a lo racional y a lo manifiesto.

Por último, la legitimidad del uso de metodología cualitativa y específicamente de los medios artísticos en investigación proviene de dos fuentes:

1. Desde la rama de la filosofía y de la ciencia que estudia la creación de conocimiento científico, la epistemología. Ésta va reconociendo que el investigador no es neutral. Ya en 1930 Cooley (en Wengrower 2001) escribió que las mejores metodologías, ya sean cuantitativas o cualitativas, provienen de científicos comprome- 
tidos activamente en investigaciones en las cuales la metodología se subordinó "al deseo ardiente de saber y comunicar algo significativo sobre la vida humana". En este caso, hablamos tanto una epistemología socio-construccionista, o una que adopta los principios de la teoría crítica

2. El descubrimiento renovado de la importancia de las emociones en el desarrollo de la persona y en la creación del conocimiento a nivel individual. La inclusión de los medios artísticos es concordante con estos dos aspectos.

\section{VALIDEZ Y CONFIABILIDAD}

Como en todo estudio sobre investigación, debemos referirnos a estos dos parámetros científicos.

Las exigencias científicas de rigurosidad, validez y confiabilidad en investigación, pueden ser satisfechas también al emplear medios artísticos. Hay diferentes medidas a tomar y argumentos a considerar. Para una información minuciosa sobre validez en investigación cualitativa y los conceptos relacionados de confiabilidad y transferibilidad se puede consultar Sandín Esteban (2000).

Confiabilidad y transferibilidad. Una de las críticas a la metodología cualitativa y a la artística es que no permite calcular la representatividad de los datos de la indagación en el total de la población, ya que no se habla estadísticamente de una muestra representativa de una población determinada. En la clínica sabemos que a menudo la investigación experimental o de laboratorio, está muy alejada de la complejidad de la realidad contextualizada y por lo tanto nos deja con más preguntas que respuestas. Al decir de Serrano cuando se refiere al estudio de caso como método: "un caso no puede representar el mundo, pero sí puede representar un mundo en el cual muchos casos se sientan reflejados". (Wengrower, 2001).

\section{ALGUNAS TÉCNICAS PARA ASEGURAR CONFIABILIDAD}

1. La sistematización y la repetición como técnicas son importantes La información obtenida a lo largo de un tiempo considerado y los fenómenos registrados reiteradamente en diferentes circunstancias o modalidades, otorgan mayor peso a las descripciones y conclusiones.

2. El investigador debe presentar un relato transparente de los pasos que siguió en su tarea, ya sea a nivel teórico como empírico.

3. Recurrir a más de un observador

4. Citas textuales del material producido en la investigación,

5. Escribir reportes que se propongan reflejar la experiencia del investigador,

6. Buscar ejemplos que contradicen la interpretación o proposición emergente.

Varias de las técnicas mencionadas hasta aquí se subsumen en lo que se ha denominado triangulación, el método por excelencia para lograr validez en investiga- 
ción cualitativa ${ }^{4}$. Ésta consiste en el empleo de técnicas variadas, incluida la redundancia de datos, explicaciones y procedimientos que desafían la información producida. El material que contradice la hipótesis inicial cuando la hay, o la tendencia que se observa en la información recogida, es un indicio de que quien lleva la indagación no está cegado por preconceptos y configura un ejemplo de la premisa de falsación de Popper. La búsqueda de repeticiones puede ser también por medio de técnicas diversas, sabiendo que no hay repeticiones idénticas. Se considera que la misma triangulación constituye una ventaja al permitir unir información complementaria y solapar medidas o testimonios del mismo fenómeno. En otro artículo mencioné que diferentes técnicas empleadas a fin de establecer triangulación, proporcionaron información complementaria y suplementaria (Wengower y Serrano 2009).

Por último, concuerdo con Hervey (2004) en que si una investigación es valorada por su aporte a una disciplina o un campo de conocimiento, en las terapias creativas en las cuales la expresión artística y el proceso creativo son fundamentales teórica y prácticamente, hemos de valernos de expresión artística y de proceso creativo. El uso de medios artísticos será evaluado en la medida en que incrementa la información y la comprensión de nuestras bases teórico-prácticas.

\section{ALGUNOS MODOS DE EMPLEO DE TÉCNICAS ARTÍSTICAS EN IN- VESTIGACIÓN}

Etnodrama o performance etnográfica: se emplea para la presentación del material de la investigación realizada, es un diálogo en el cual los actores (performers) y el público actúan en un intercambio igualitario. Los co-performers leen el texto basado en el material producido en el trabajo de campo y las entrevistas. Es una forma de presentación de los temas que afloraron en la investigación de evaluación de algún centro, una comunidad, un modelo de intervención, etc. Favorece la reflexión entre quienes llevaron a cabo la evaluación y los participantes del ente evaluado. Mienczakowski (2001) reconoce la contribución del proceso artístico para la introspección o lo que él denomina el auto-descubrimiento de los sujetos, el proceso de integración de lo interno y externo y de los niveles emocional y estético.

El teatro se usa como un medio de información, transmisión de la información producida en la etnografía, verificación, diálogo con los informantes, difusión y educación. Añade otro paso y otro dispositivo al modo tradicional de presentación escrita. El etnodrama emplea elementos de cine, video, performance y teatro. No sólo quiere mostrar que los límites entre realidad y ficción no son claros, sino que aspira a educar y liberar, empoderar (empower), emancipar, constituirse en una forma de reflexión para los sujetos. Se ha empleado este modelo con el propósito de difuminar los límites entre proveedores y consumidores de salud, por eso la cons-

\footnotetext{
${ }^{4}$ Recordemos que por lo general los medios artísticos se incluyen en esta metodología, aunque no necesariamente, se puede pensar en análisis cuantitativo del material artístico o en la integración de métodos cualitativos y cuantitativos.
} 
tante validación y diálogo. A diferencia del modelo del Teatro del Oprimido de Boal, el etnodrama no persigue producir algo estético. Esta técnica se usó en el campo de la salud pública y como metodología de prevención de suicidios.

Por este medio, los investigadores devuelven a la comunidad el conocimiento recibido, con la intención de manifestar que la influencia es mutua, las personas en sus diferentes roles son co-performers, co-actores y co-agentes en la vidas de los otros. El etnodrama hace accesible el producto de la investigación a la audiencia, como método relacionado con la etnografía da sentido a los aspectos visuales y los objetos de su comunidad, el servicio asistencial en el cual están involucrados, etc. O sea que estamos frente a un ejemplo que añade otra forma y otro paso al de escritura de la investigación. Por un lado, se agrega la presentación teatral a la presentación escrita y se comparte la información con la comunidad o grupo con quienes se investigó. Como ya se introdujo arriba en este artículo, esta es otra manifestación del paradigma interpretativo y de su conceptualización del investigador no neutral, participante en la realidad que estudia y de que las personas no son objeto de estudio, sino sujetos con quienes se estudia. Desde los cambios epistemológicos en investigación y al admitir que no hay científico neutral, que toda indagación modifica y por tanto se investiga con los sujetos y no a los sujetos, que la información producida debe redundar en beneficio de la comunidad, la cual a su vez no sólo reflexiona sino que juzga el material, el último paso es comunicarlo a y con la colectividad o los sujetos. El etnodrama es un modelo combinado entre la etnografía y las artes escénicas. Se ubica dentro de las líneas de teatro para la transformación social, pero más allá de demandas artísticas, es un intento de producir crítica cultural. El medio artístico se emplea a fin de convocar respuestas emocionales y un conocimiento implicado en ellas en la audiencia, parte de la cual ha sido sujeto de la investigación.

Danzando la información: también se emplea la danza para presentar los resultados de investigaciones. No sólo en nuestra área, sino también en ciencias naturales y sociales y en el ámbito de la educación, como forma complementaria al texto escrito. En el primer caso, hace ya varios años que en el mundo anglosajón hay una competición denominada Dance your Ph.D. ${ }^{5}$ Para el área de educación ver Bagley y Cancienne, 2001.

Otra modalidad es la que durante los años que dirigí el master en Terapia a través del Movimiento y la Danza en la Universidad de Barcelona (cerrado ya) instituimos: la composición de una danza al final de cada curso como modo de síntesis e integración del proceso de estudios y de formación. Sé que algunos otros programas de formación lo emplean también. La composición de una danza personal basada en una serie de puntos a reflexionar entregada a las estudiantes, además del texto escrito, constituyó parte del proceso de cierre del curso que favoreció la integración de los aspectos cognitivos, emocionales y de movimiento. La presentación de la danza

\footnotetext{
${ }^{5}$ Se puede ver la danza ganadora en 2011 y un texto de explicación en:

http://news.sciencemag.org/sciencenow/2011/10/dance-your-phd-winner-announced.html
} 
en el grupo facilitaba el reconocimiento del proceso personal y era consecuente con el trabajo llevado a cabo en los estudios en el cual el grupo de estudiantes se concebía como una fuente más de aprendizaje. En este caso estamos hablando del uso de la danza como parte de un sistema de métodos de análisis y de integración del proceso de formación. Se trata de investigación como reflexión e integración de un proceso personal de formación, que puede servir además para que los involucrados, estudiantes y docente, puedan evaluar el impacto de la tarea realizada.

\section{EXPLORACIÓN DE EXPERIENCIAS Y TEMAS RELACIONADOS CON GÉNERO POR MEDIO DE ARTES ESCÉNICAS}

B. Allegranti (2009) basándose en la teoría feminista de J. Butler y su concepto de performatividad, llevó a cabo un proyecto en el cual integró el trabajo en danzamovimiento y la producción de videos cortos para explorar la deconstrucción y reconstitución de la performatividad de la sexualidad y del género de los participantes nueve participantes. Considero que este es un ejemplo de integración entre práctica e investigación.

Empleo de técnicas de dramaterapia para investigación psicosocial. ${ }^{6}$

Durante varios años conduje grupos con niños, adolescentes y adultos en Jerusalén para explorar y tratar las actitudes ante los inmigrantes compañeros de clase o de escuela; también dirigí talleres para estudiar las actitudes de los maestros de esos alumnos y con trabajadores sociales que trabajaban con población inmigrante. Este país, instituido en 1948, tres años después del fin de la catástrofe humana que significó la segunda guerra mundial, favorece oficialmente la inmigración de personas de origen judío, sin embargo, la integración social de los mismos presenta dificultades como en cualquier sociedad (Wengrower, 1992, 2001, Wengrower y Serrano 2009).

Los criterios que orientaron la elección del método artístico de investigación basado en técnicas teatrales y la imaginería guiada fueron los siguientes:

Definición y demandas del rol implican la contraindicación de determinadas técnicas.

Dalal (1993) observó que en las últimas décadas del siglo XX en los círculos psicoterapéuticos se hacía más fácil hablar de fantasías incestuosas que de prejuicios interétnicos, dada la presión de lo "políticamente correcto". Al trabajar con niños y adolescentes y especialmente con los profesionales mencionados, agentes de endoculturación, la aproximación directa hacia sus actitudes o imágenes creadas de los inmigrantes, fue considerada ineficaz. Hubiese implicado crear un conflicto abierto entre mundo interno y externo por así decirlo, o entre exigencias de rol, ideología oficial y el nivel individual-personal. Las técnicas de dramaterapia fueron un tipo de procedimiento elegido para acceder por medio de la metáfora y la distancia estética (Landy, 1983) a esas imágenes y a los aspectos emocionales de las actitudes. En el caso del trabajo con niños, desdeñé la técnica del diferencial semántico

\footnotetext{
${ }^{6}$ Para una lectura más detallada y extensa, ver Wengrower 1995, 2001, 2002).
} 
tan empleada para la valoración de actitudes ya que su estructura favorece el pensamiento dicotómico, tan afín al prejuicio (Wengrower y Serrano 2009) ${ }^{7}$

Otros factores de orden ético y psicológico fueron considerados.

Niveles de exposición tolerables.

La mayoría de los profesionales participantes se veían diariamente o con alta frecuencia. Esto demandaba emplear técnicas que permitieran simultáneamente la expresión auténtica e íntima cuidando los límites y distancias interpersonales, grado de exposición, personal, etc. Las técnicas elegidas fueron la imaginación guiada y los juegos dramáticos. El trabajo en danza movimiento (el medio que más manejo y por el que me defino) requiere un proceso más largo de caldeamiento del grupo y propicia aspectos más regresivos o íntimos, que podrían elevar el nivel de inquietud.

En niños y adolescentes también es importante mantener un nivel de ansiedad bajo. Con ellos utilicé técnicas de dramaterapia.

Relación eficaz entre técnica y tiempo destinado a la investigación-intervención.

Cuando la decisión de investigar un tema está tomada, el criterio de eficacia del método a emplear es otro de los factores a sopesar. Los grupos de niños o adolescentes se encontraban durante ocho o catorce sesiones semanales y estaban formados por participantes de ambos géneros, los talleres para adultos consistían en una reunión de dos a tres horas, hubo formatos diferentes. Rápidamente observé que las técnicas dramáticas eran más fácilmente aceptadas que las de danza/movimiento por los niños, los muchachos (género masculino) y por los adultos en general ${ }^{8}$. Como dije arriba, estas técnicas demandan un proceso más largo de caldeamiento y pueden convocar emociones o temas no necesariamente conectados con la pregunta de la investigación y que se relacionan con la técnica. Creo que sería interesante llevar a cabo un proyecto que compare dos grupos empleando uno técnicas dramáticas y el otro, técnicas de danza/movimiento.

Descripción de los grupos de niños

La participación en los grupos es voluntaria, contando como promedio con ocho participantes. El acento está puesto en la tarea convocante como en el modelo de los grupos operativos (Pichón Riviere, 1960) y consiste en crear historias y escenas sobre las amistades entre pares. La conductora ofrece imágenes, metáforas o analogías a trabajar teatralmente, se incluyen intervenciones que se relacionan con los fenómenos de aceptación de la diferencia interpersonal o intragrupal de modo simbólico, con el establecimiento (o no) de relaciones nuevas, competencia, etc. El objeto de la indagación que se presentó a los niños fue el de las amistades entre

7 "El diferencial semántico es un procedimiento destinado a medir la significación que tienen ciertos objetos, hechos, situaciones o personas para los encuestados. Para establecer el "diferencial semántico" de un objeto, situación, hecho o persona, se proponen pares de adjetivos contrapuestos, y se pide al encuestado que sitúa la cercanía a cada extremo del par en una escala de 7 grados". (Roldán Carmona y Orozco Durán, 2006)

${ }^{8}$ A pesar de que la sociedad israelí es relativamente abierta en lo que se refiere a cuerpo, comparándola con la cultura latina, es bastante más cerrada. El empleo mediatizado del cuerpo en teatro es por tanto más fácilmente aceptable que la danza o el movimiento expresivo. 
pares, sin mencionar las categorías de inmigrantes y nativos. Esta formulación es simultáneamente verdadera y previene la posibilidad de que el mismo método de investigación cree un estigma.

La sesión consta de cuatro partes. Primero, calentamiento psicofísico y del grupo, luego preparación de historias-escenas relacionadas con el tema en subgrupos organizados espontáneamente, tercero, presentación de las historias ante el resto de compañeros y por último reflexión grupal sobre cada una de las creaciones. En esta parte de la sesión se comenta lo observado, se comparten las emociones y asociaciones despertadas, las conexiones que establecen con sus propias experiencias o conocimientos en su vida social.

La imagen propuesta para iniciar el proceso de indagación y creación de historias es el arribo de alguien o algo nuevo a un lugar en el cual hay otros de la misma categoría, por lo general se trata de algún objeto, planta o flor que arriba a un estante, un jardín. Una cacerola nueva viene o es traída a una tienda, llega o aparece un rosal nuevo, etc.

Otra técnica empleada es el monólogo subsiguiente de los personajes y las preguntas posteriores que el público, el resto del grupo, formula a dicho personaje acerca de las vivencias dramatizadas en la historia o escena.

El material producido con estas técnicas aportó información acerca de las ansiedades que despierta el inmigrante simbolizado en el nuevo o el recién llegado, las tácticas que emplean los niños de la clase o los subgrupos que la componen ante su arribo e inclusión impuesta por la escuela. Aparecieron contenidos preconscientes e inconscientes que pudieron ser elaborados metafóricamente primero y luego verbal y cognitivamente aportando una mayor comprensión a los niños de su situación. El material coincidió con análisis teóricos escritos desde la sociología o la antropología y permitió configurar una descripción compleja o densa (Wengrower y Serrano, 2009) de las relaciones de pares inmigrantes y nativos en entornos escolares. Si en el etnodrama el teatro es empleado fundamentalmente para los pasos finales de la investigación, en este modelo es parte de las modalidades de producción de información, o lo que en el paradigma positivista se llama recolección de datos.

\section{CONCLUSIÓN}

Este artículo presentó la necesidad de incrementar la investigación en las terapias creativas, la posibilidad de hacerlo empleando metodología cualitativa y especialmente medios artísticos. Luego se expusieron algunos métodos a fin de compartir ejemplos de la integración del arte y la investigación y también sugerir la ampliación de nuestro quehacer a otros campos.

\section{REFERENCIAS BIBLIOGRÁFICAS}

ALLEGRANTI, B. (2009): Embodied performances of sexuality and gender: A feminsit approach to Dance Movement Therapy and performance practice. Body, Movement and Dance in Psychotherapy, 4, 1, 17-31. 
BAGLEY, C., CANCIENNE, M.B. (2001): Educational Research and Intertextual Forms of (Re) Presentation: The Case for Dancing the Data. Qualitative Inquiry 7: 221-237.

CHAIKLIN, H. Y CHAIKLIN, S. (2004): The case study. En R. Cruz y C. Berrol (Eds.): Dance/movement therapists in action: A working guide to research options. Spingfield Illinois, Charles Thomas. 69-91.

CRUZ, R. Y BERROL C. (2004): Dance/movement therapists in action: A working guide to research options. Spingfield Illinois, Charles Thomas.

DALAL, F. (1993): 'Race' and Racism: An Attempt to Organize Difference. Group Analysis, 26:277-293.

DETWEILER, (2000): A guide to treatment that works. APA Review of books, 45(2): 148-151.

EISER, R. (1989): Psicología social. Actitudes, cognición y conducta social. Madrid, Pirámide.

GREEN, J., N. BRITTEN (1998): Qualitative research and evidence based medicine British Medical Journal Vol. 316, 1230-1232.

HERVEY, L. (2004): Artistic Inquiry in Dance/Movement Therapy. En R. Cruz, R. y C. Berrol, (Eds), Dance/movement therapists in action: A working guide to research options. Springfield, IL, US: Charles C Thomas. 181-205.

HERVEY, L. (2008: Cómo alentar la investigación en DMT. En H. Wengrower y S. Chaiklin (coords.): La Vida es danza. El arte y la ciencia de la Danza Movimiento Terapia. Barcelona, Gedisa. 355-370

LANDY, R. (1983): The Use of Distancing in Drama-Therapy. En The Arts in Psychotherapy, vol. 10:175-185.

MC NIFF, S. (1998): Art-based research. Londres, Jessica Kingsley.

MEEKUMS, B. (2010): Moving towards evidence for dance movement therapy: Robin Hood in dialogue with the King. Arts in Psychotherapy, 37, 1, 35-41.

MIENCZAKOWSKI, J. (2001): Ethnodrama: performed research- Limitations and potential. En P. Atkinson, Coffey, A., Delamont, S. Lofland, J., Lofland, L. (eds.): Handbook of Ethnography. Londres, Sage. 468-476.

PICHON RIVIERE, E. (1960): Técnica de los grupos operativos. Acta Neuropsiquiátrica Argentina, 6, 107-120.

REASON, P. (Ed.) (1994 a): Participation in Human Inquiry. Londres, Sage Publications. 
ROLDÁN CARMONA, OROZCO DURÁN, J.O. (2006): El diferencial semántico. Universidad San Marcos. http://www.espacioblog.com/myfiles/quirosleiva. Extraído 12-5-09

SANDÍN ESTEBAN, M.P. (2000): Criterios de validez en la investigación cualitativa: de la objetividad a la solidaridad. Revista de Investigación Educativa, 2000, Vol. 18, n. $^{\circ} 1,223-242$

WENGROWER, H. (1992): Relaciones entre niños inmigrantes y nativos. Tesis no publicada de master. Univ. Hebrea. Jerusalén.

WENGROWER, H. (1994): Guided Imagination: A workshop model for teachers working with new immigrants. En: Theory in relation to practice: Professional intervention among immigrants from the former Soviet Union. Jerusalén, Universidad Hebrea, 114-122

WENGROWER, H. (1995): "The tools of Expressive Therapies in the social field: Immigrants and the host society". En: Conference Proceedings, Third European Arts Therapies Conference, Ecarte, Ferrara, Italia. 183-188.

WENGROWER, H. (2001): Yo-nosotros, tú-vosotros. Estudio psicosocial de la relaciones entre niños inmigrantes y nativos en el marco educativo. http://www.tdx.cat/TDX-0506102-111719.

WENGROWER, H. (2008): El proceso creativo y la actividad artística por medio de la danza y el movimiento. In H. Wengrower, S. Chaiklin (eds) (2008).: La Vida es Danza. El Arte y la Ciencia de la Danza Movimiento Terapia. Barcelona, Gedisa.

WENGROWER, H. (2009): Dance Then and Now; in History and in the Individual. Why Dance in Therapy. In Scoble, S., Ross, M., Lapoujade, C. (eds.): Arts in Arts Therapies: A European Perspective. Plymouth, University of Plymouth Press.

WENGROWER, H. Y SERRANO, J. (2009), Técnicas inmigrantes en el país de las ciencias sociales. Informe del empleo de técnicas especiales en una investigación con niños inmigrantes y nativos. Arteterapia, Papeles de arteterapia y educación artística para la inclusión social. Publicaciones de la Univ. Complutense de Madrid. Vol. IV, 111-136 